\title{
Facile synthesis of nickel cobaltite quasi-hexagonal nanosheets for multilevel resistive switching and synaptic learning applications
}

\author{
Tukaram D. Dongale ${ }^{1,2}$, Atul C. Khot', Ashkan Vakilipour Takaloo ${ }^{1}$ and Tae Geun Kim (1)
}

\begin{abstract}
High-density memory devices are essential to sustain growth in information technology (IT). Furthermore, braininspired computing devices are the future of IT businesses such as artificial intelligence, deep learning, and big data. Herein, we propose a facile and hierarchical nickel cobaltite (NCO) quasi-hexagonal nanosheet-based memristive device for multilevel resistive switching (RS) and synaptic learning applications. Electrical measurements of the Pt/ NCO/Pt device show the electroforming free pinched hysteresis loops at different voltages, suggesting the multilevel RS capability of the device. The detailed memristive properties of the device were calculated using the timedependent current-voltage data. The two-valued charge-flux properties indicate the memristive and multilevel RS characteristics of the device. Interestingly, the Pt/NCO/Pt memristive device shows a compliance current (CC)dependent RS property; compliance-free RS was observed from $10^{-2}$ to $10^{-4} \mathrm{~A}$, and the compliance effect dominated in the range of $10^{-5}-10^{-6} \mathrm{~A}$. In CC control mode, the device demonstrated three resistance states during endurance and retention measurements. In addition, the device was successful in mimicking biological synaptic properties such as potentiation-depression- and spike-timing-dependent plasticity rules. The results of the present investigation demonstrated that solution-processable NCO nanosheets are potential switching materials for high-density memory and brain-inspired computing applications.
\end{abstract}

\section{Introduction}

The increasing demand for miniaturized electronic devices for information processing has triggered innovations in nonvolatile memory technology. Traditional charge-storage-based memory devices are facing serious technological limitations for further miniaturization and are unable to address current technological demands. The bottleneck in the operation of charge-based memory devices arises from their inherent structural design and current leakage issue ${ }^{1}$. These problems can be overcome by utilizing a principle that is scalable in the sub-10-nm range, tolerant to leakage current, and compatible with

\footnotetext{
Correspondence: Tae Geun Kim (tgkim1@korea.ac.kr)

${ }^{1}$ School of Electrical Engineering, Korea University, Anam-ro 145, Seongbuk-gu, Seoul 02841, Republic of Korea

${ }^{2}$ School of Nanoscience and Biotechnology, Shivaji University,

Kolhapur 416 004, India
}

conventional silicon-based fabrication technology ${ }^{2}$. The resistance switching (RS) effect promises the abovementioned features along with 3D integration ${ }^{3}$, highdensity memory storage ${ }^{4}$, and in-memory computing capabilities ${ }^{5}$. The exponential growth in artificial intelligence, machine learning, and big data in the last several years has affected information processing and data storage devices $^{6}$. These technologies demand high-density memory devices to store and retrieve data for each application request, which enables them to provide a real-time experience to end users.

The RS effect can be tuned to obtain multiple resistance states, which allows for the development of a high-density memory device without compromising scaling and power consumption $^{7}$. In addition, multilevel RS is useful in mimicking biological synaptic properties, helping to realize brain-like computing capabilities ${ }^{8}$. To mimic synaptic 
properties, the device should exhibit an analog RS effect. The RS of the device can be easily engineered to achieve analog memory by selecting a proper active switching layer material. In the past, various kinds of materials have demonstrated analog memory properties. These materials include but are not limited to $\mathrm{TaO}_{\mathrm{x}}{ }^{9}, \mathrm{SrTiO}_{3}{ }^{10}, \mathrm{BiFeO}_{3}{ }^{11}$, $\mathrm{ZnO}^{12}, \mathrm{WO}_{3}{ }^{13}, \mathrm{TiO}_{2}{ }^{14}$, and $\mathrm{NiO}^{15}$. These conventional materials have problems such as nonuniformity in switching events, a high electroforming voltage, and a low device yield ${ }^{16}$. Therefore, new materials are being investigated by academia and industry for the best possible RS operation. Nickel cobaltite, often referred to as NCO, is a binary metal oxide that is popular for energy storage device applications owing to its rich electrochemical properties ${ }^{17}$. NCO shows excellent redox reaction capability and electrical conductivity owing to the availability of both ions in its structure (nickel and cobalt) ${ }^{18}$. The abundance of oxygen vacancies in the NCO spinel-type structure can help to tune the conductive filament in RS devices $^{19}$. NCO is a low-cost material, is easily available, and possesses low toxicity ${ }^{20}$. Solution-processable NCO often shows a quasi-hexagonal nanostructure morphology and is a thermodynamically stable morphology for electronic device applications ${ }^{21}$. In addition, the porous nature of the quasihexagonal nanostructure provides excellent charge transport pathways ${ }^{22}$, which can be employed to obtain excellent RS properties from the device. These features make NCO a natural choice for RS applications.

Multilevel RS is a unique approach for creating a highdensity nonvolatile memory device. Interestingly, the active switching layer plays an important role in multilevel RS. In most of the reported work, filamentary RS, composed of oxygen vacancies, helps to achieve multiple resistance states within the same device ${ }^{23}$. In general, the size of the conductive filament can be tuned by controlling the compliance current $(\mathrm{CC})$, resulting in multiple resistance states ${ }^{24}$. In another approach, the gap between the tip of the conductive filament and the bottom electrode can be varied by the application of different RESET voltages $^{25,26}$.

Herein, we report on the synthesis, characterization, and RS properties of a Pt/NCO/Pt device. To the best of the authors' knowledge, this is the first report on the RS of NCO nanomaterials. The RS of the $\mathrm{Pt} / \mathrm{NCO} / \mathrm{Pt}$ device was correlated with memristive switching and demonstrated double-valued charge-flux characteristics. A detailed investigation of the voltage and CC-dependent RS properties of the $\mathrm{Pt} / \mathrm{NCO} / \mathrm{Pt}$ memristive device was carried out. By controlling the $\mathrm{CC}$, three distinct resistance states were achieved during endurance and retention measurements. Furthermore, the analog memory properties of the $\mathrm{Pt} / \mathrm{NCO} / \mathrm{Pt}$ memristive device were used to mimic biosynaptic properties.

\section{Experimental section}

\section{Synthesis of NCO nanomaterial}

The NCO nanomaterial was synthesized using the coprecipitation method. For the synthesis, $0.0015 \mathrm{~mol}$ of $\mathrm{NiCl}_{2} \cdot 6 \mathrm{H}_{2} \mathrm{O}$ (Sigma Aldrich) and $0.0030 \mathrm{~mol}$ of $\mathrm{CoCl}_{2} \cdot 6 \mathrm{H}_{2} \mathrm{O}$ (Sigma Aldrich) were dissolved separately in two beakers containing $30 \mathrm{ml}$ of deionized (DI) water and stirred for $15 \mathrm{~min}$. The solution containing nickel salt was added dropwise to the other solution (cobalt salt) and stirred for $15 \mathrm{~min}$ to form an intermediate solution. Then, $0.010 \mathrm{~mol}$ of $\mathrm{NaOH}$ (Sigma Aldrich) was dissolved in another beaker with constant stirring for $10 \mathrm{~min}$ and added dropwise to the intermediate solution using a burette. The mixture was stirred for $30 \mathrm{~min}$ to achieve complete consumption of the reactants. The obtained precipitate was centrifuged at 4000 RPM for $10 \mathrm{~min}$ with DI water and ethanol twice each and dried at $70^{\circ} \mathrm{C}$ for $6 \mathrm{~h}$. Finally, the powder was crushed and calcined at $450{ }^{\circ} \mathrm{C}$ for $3 \mathrm{~h}$ at a $5^{\circ} \mathrm{C} / \mathrm{min}$ ramp rate.

\section{Fabrication of $\mathrm{Pt} / \mathrm{NCO} / \mathrm{Pt}$ RS device}

To fabricate the active switching layer, $0.250 \mathrm{~g}$ of $\mathrm{NCO}$ nanomaterial was initially mixed with $0.250 \mathrm{~g}$ of ethylcellulose and $2 \mathrm{~mL}$ of ethanol. To the prepared mixture, $2 \mathrm{~mL}$ of alpha-terpineol was added, and the entire mixture was stirred for $30 \mathrm{~min}$. The final product was spin-coated on a clean Pt $(50 \mathrm{~nm}) / \mathrm{Ti}(50 \mathrm{~nm}) / \mathrm{SiO}_{2} / \mathrm{Si}$ substrate. The thickness of the NCO layers varied in the following order: $100,250,500$, and $750 \mathrm{~nm}$. To obtain a thickness of $100 \mathrm{~nm}$, the paste was spin-coated at $3000 \mathrm{RPM}$ for $30 \mathrm{~s}$. This procedure was repeated three, five, and eight times to obtain 250, 500, and $750 \mathrm{~nm}$ thicknesses. After spincoating, the film was annealed at different temperatures $\left(150,250,350,450\right.$, and $\left.550^{\circ} \mathrm{C}\right)$ for $1 \mathrm{~h}$. These annealing temperatures remove the binder and other dispersant agents from the spin-coated film. In the present case, the device with $500 \mathrm{~nm}$ thickness and a $350{ }^{\circ} \mathrm{C}$ annealing temperature exhibited better RS properties than the other devices. A radio-frequency sputtering system along with a shadow mask was used to deposit the top platinum (Pt) electrode (thickness: $\sim 100 \mathrm{~nm}$ and diameter $\sim 200 \mu \mathrm{m}$ ).

\section{Results and discussion}

Figure 1a, b show field-emission scanning electron microscopy (FESEM) (LYRA3, TESCAN) and highresolution FESEM images, respectively, of the NCO nanomaterials. The coprecipitation synthesis technique generally helps to form a hierarchical nanostructure ${ }^{27}$. In the present case, a hierarchical quasi-hexagonal nanosheet morphology was achieved by a facile and low-cost synthesis technique. The quasi-hexagonal nanosheets were composed of smaller crystallites, as observed from the highresolution FESEM image (Fig. 1b). The FESEM images were further analyzed using ImageJ software. The statistical 

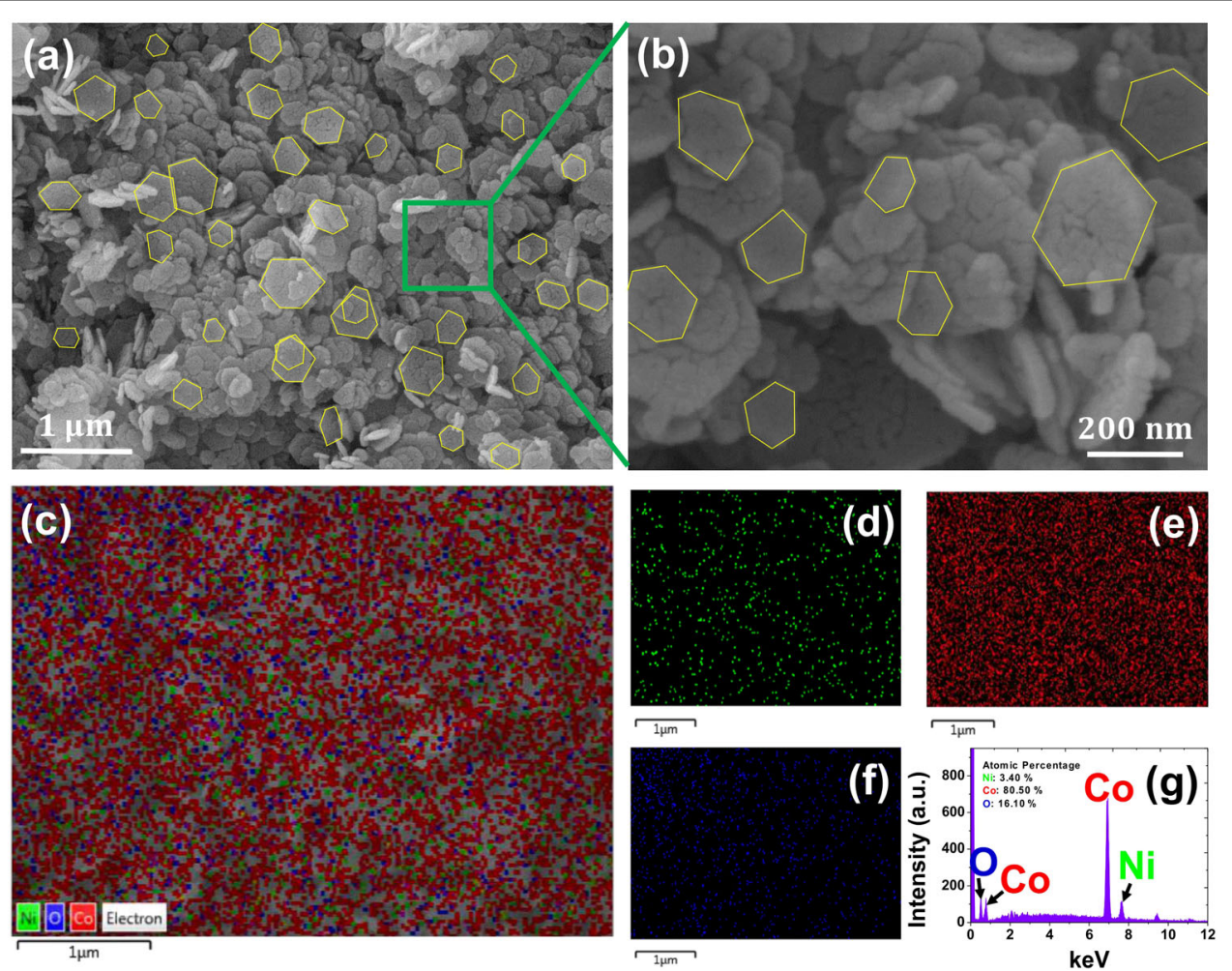

Fig. 1 Morphological and compositional characterizations of the quasi-hexagonal NCO nanosheets. a FESEM and $\mathbf{b}$ high-resolution FESEM images of quasi-hexagonal NCO nanosheets. c Energy-dispersive X-ray spectroscopy (EDS) mapping of quasi-hexagonal NCO nanosheets and corresponding $\mathbf{d} \mathrm{Ni}$, e Co, and $\mathbf{f} \mathrm{O} . \mathbf{g}$ EDS spectrum of quasi-hexagonal NCO nanosheets.

distributions of the side length and thickness of the hexagonal nanosheets are shown in Fig. S1a, b, respectively. Statistical measures such as the mean, standard deviation, and coefficient of variation suggest that the length and thickness of the quasi-hexagonal nanosheets are uniform in nature. Interestingly, the quasi-hexagonal nanosheets of NCO were porous. The Brunauer-Emmett-Teller (BET) and Barrett-Joyner-Halenda (BJH) techniques were used to determine the specific surface area and pore size distribution of $\mathrm{NCO}$ by $\mathrm{N}_{2}$ adsorption/desorption isotherms at $77 \mathrm{~K}$, as shown in Fig. S2a, b, respectively (ASAP 2020+, Micromeritics Instruments). The surface area was found to be $47.81 \mathrm{~m}^{2} \mathrm{~g}^{-1}$, whereas the mean pore diameter was $17.70 \mathrm{~nm}$, suggesting the mesoporous nature of the synthesized NCO nanomaterial. In addition, the degree of porosity was found to be $2.35 \%$. Porous NCO nanomaterials are very helpful in creating good charge transport pathways $^{28}$.

Figure 1c presents the energy-dispersive $\mathrm{X}$-ray spectroscopy (EDS) result for the quasi-hexagonal NCO nanosheets, and the corresponding mapping results for $\mathrm{Ni}, \mathrm{Co}$, and $\mathrm{O}$ are shown in Fig. 1d, e, and f, respectively. The mapping results assert the presence of $\mathrm{Ni}, \mathrm{Co}$, and $\mathrm{O}$ in the sample. Furthermore, the EDS spectrum of the quasi-hexagonal $\mathrm{NCO}$ nanosheets was recorded to understand the quantitative distribution of the component elements, as shown in Fig. 1g. The elemental concentrations (atomic \%) of $\mathrm{Ni}, \mathrm{Co}$, and $\mathrm{O}$ were $3.40 \%$, $80.50 \%$, and $16.10 \%$, respectively, as observed from the EDS results. The X-ray diffraction (XRD, SmartLab, Rigaku) pattern of the NCO nanomaterial is shown in Fig. S3. The diffraction peaks of NCO match well with JCPDS card No. 20-0781, suggesting the formation of a cubic structure $^{29}$. The experimental XRD data were further analyzed to obtain insight into the NCO nanomaterial. The crystallite size, strain (lattice defect), the lattice constant, and \% crystallinity of the NCO nanomaterial were found to be $19.62 \mathrm{~nm}, 1.4 \times 10^{-3}, 8.09 \mathrm{~nm}$, and $68.73 \%$, respectively. The results suggested that nonstoichiometric NCO was formed by the coprecipitation synthesis technique used in this work ${ }^{30}$.

X-ray photoelectron spectroscopy (XPS) (X-Tool, ULVAC-PHI) was utilized to understand the elemental composition of the NCO nanomaterial. The survey spectrum of the NCO nanomaterial is shown in Fig. 2a. Intense peaks of Ni 2p, Co 2p, and O 1s were observed in the survey spectrum. The peak at $284.5 \mathrm{eV}$ (C 1s) reveals the presence of carbon in the sample arising from air contact with the NCO sample. The narrow-scan spectrum of $\mathrm{Ni} 2 \mathrm{p}$ shows two spin-orbit doublets at binding energies 

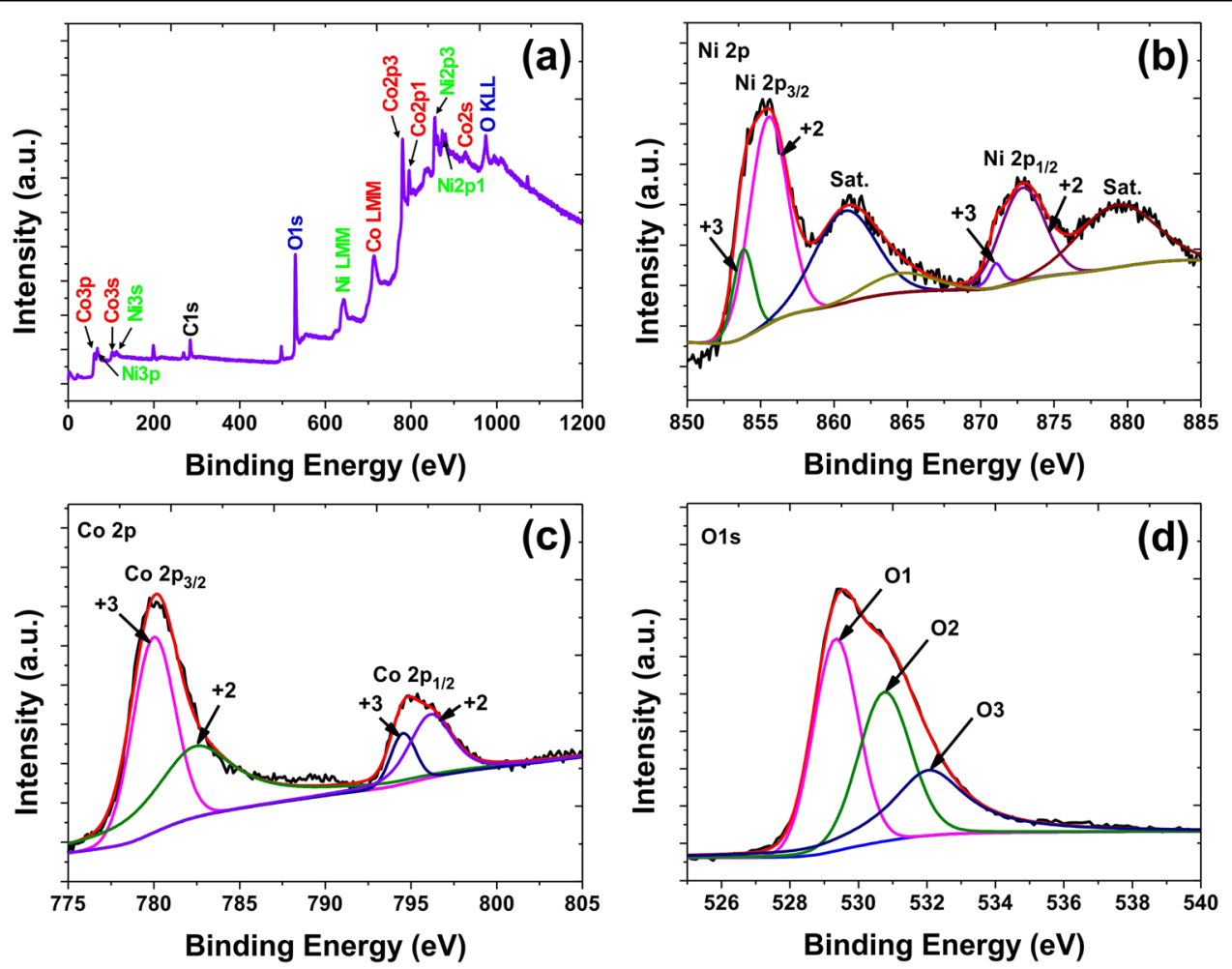

Fig. 2 XPS characterization of quasi-hexagonal NCO nanosheets. a Survey spectrum and narrow-scan/core-level spectra of (b) $\mathrm{Ni}$, (c) Co, and (d) $\mathrm{O}$.

of 855.3 and $872.9 \mathrm{eV}$, corresponding to $\mathrm{Ni} 2 \mathrm{p}_{3 / 2}$ and $\mathrm{Ni}$ $2 \mathrm{p}_{1 / 2}$, respectively (Fig. $2 \mathrm{~b}$ ). The results confirm that two oxidation states, i.e., $\mathrm{Ni}^{3+}$ and $\mathrm{Ni}^{2+}$, were present in the sample $^{31}$. Two satellite peaks were observed at binding energies of 861.1 and $879.6 \mathrm{eV}$. Figure 2c shows the corelevel spectrum of Co 2p. Two spin-orbit doublets, i.e., Co $2 \mathrm{p}_{3 / 2}$ and Co $2 \mathrm{p}_{1 / 2}$, were observed at binding energies of 780.1 and $794.8 \mathrm{eV}$, respectively. These two peaks (Co $2 \mathrm{p}_{3 /}$ 2 and Co $2 \mathrm{p}_{1 / 2}$ ) were deconvoluted into an additional two peaks. The peaks observed at 777.9 and $794.5 \mathrm{eV}$ corresponded to the $\mathrm{Co}^{3+}$ oxidation state. The peaks observed at 782.5 and $796.2 \mathrm{eV}$ corresponded to the $\mathrm{Co}^{2+}$ oxidation $\operatorname{state}^{32}$. The core-level spectrum of $\mathrm{O} 1 \mathrm{~s}$ is shown in Fig. $2 \mathrm{~d}$. The spectrum of $\mathrm{O} 1 \mathrm{~s}$ was found to be asymmetric and was therefore deconvoluted into three Gaussian peaks. These peaks were observed to occur at $529.3 \mathrm{eV}\left(\mathrm{O}_{1}\right)$, $530.7 \mathrm{eV}\left(\mathrm{O}_{2}\right)$, and $532.1 \mathrm{eV}\left(\mathrm{O}_{3}\right)$. The peak observed at the $\mathrm{O}_{1}$ position was ascribed to $\mathrm{O}-\mathrm{Co} / \mathrm{Ni}$ bonds and lattice oxygen $^{33}$. An oxygen vacancy-related peak was observed at the $\mathrm{O}_{2}$ position ${ }^{34}$. The $\mathrm{O}_{3}$ peak corresponded to chemisorbed surface water and surface oxygen ${ }^{35}$.

The RS properties of the Pt/NCO/Pt device were measured using a Keithley 4200 parameter analyzer equipped with a probe station. For electrical measurements, the voltage was applied to the top electrode, while the bottom electrode was grounded. We preliminarily investigated the thickness-dependent RS properties of the $\mathrm{Pt} / \mathrm{NCO} / \mathrm{Pt}$ device. For this study, we varied the thickness of the NCO layer $(100,250,500$, and $750 \mathrm{~nm})$, and current-voltage (I-V) properties were recorded for each device, as shown in Fig. S4a. The thickness of the switching layers was measured using a surface profiler (Alpha-Step IQ), as shown in Fig. S4b. For the lower-thickness devices (100 and $250 \mathrm{~nm}$ ), poor RS properties were observed. This may be due to the smaller number of hexagonal nanosheets packed in a given memory cell area. Good RS performance was observed for the 500-nm-thick device owing to the optimized packing density of the hexagonal nanosheets in the memory cell. The RS properties were found to deteriorate for the 750-nm-thick device. In addition, we checked the performance of the optimized device $(500 \mathrm{~nm})$ by annealing the spin-coated layer at different temperatures $\left(150,250,350,450\right.$, and $\left.550^{\circ} \mathrm{C}\right)$. The devices annealed at 450 and $550^{\circ} \mathrm{C}$ showed multiple cracks and discontinuities in the switching layer. Therefore, I-V curves were not measured for these devices. The I-V properties of the devices annealed at 150,250 , and $350{ }^{\circ} \mathrm{C}$ are shown in Fig. S4c. Herein, no RS response was observed for the devices annealed at 150 and $250^{\circ} \mathrm{C}$. This is because the binder and other dispersant agents could not be removed from the spin-coated film at these temperatures $^{36}$. On the other hand, the device annealed at 


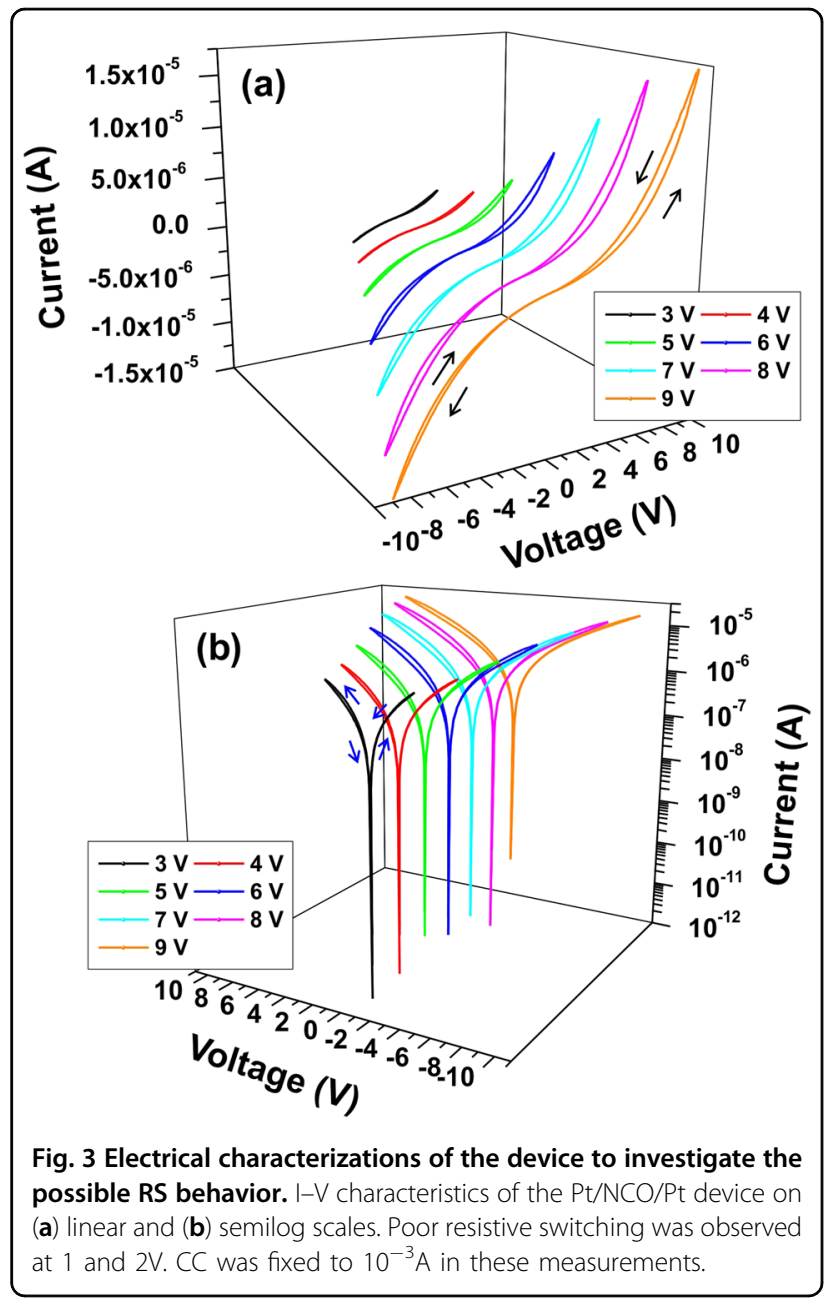

$350^{\circ} \mathrm{C}$ showed good RS properties, and hence, it was used for further investigations.

Figure $3 \mathrm{a}, \mathrm{b}$ show the current-voltage $(\mathrm{I}-\mathrm{V})$ behavior of the $\mathrm{Pt} / \mathrm{NCO} / \mathrm{Pt}$ device on linear and semilog scales, respectively. The switching direction is indicated by arrows. We applied different $\pm V_{\text {STOP }}$ voltage excitations $( \pm 3$ and $\pm 9 \mathrm{~V})$ to understand the device switching mechanism and demonstrate multilevel RS behavior. CC was fixed at $10^{-3} \mathrm{~A}$ was fixed for this investigation. During the I-V measurements, it was observed that the device showed poor RS behavior at $\pm 1 \mathrm{~V}$ and $\pm 2 \mathrm{~V} \pm \mathrm{V}_{\text {STOP }}$ voltage excitations. Stochastic RS was observed when the device was subjected to voltages greater than $\pm 9 \mathrm{~V}$. The switching direction of the device was counterclockwise in nature.

The $\mathrm{Pt} / \mathrm{NCO} / \mathrm{Pt}$ device showed voltage-dependent RS. In other words, the RS properties of the device improved as the applied $\pm \mathrm{V}_{\text {STOP }}$ voltage excitation increased from \pm 3 to $\pm 9 \mathrm{~V}$. The $\mathrm{Pt} / \mathrm{NCO} / \mathrm{Pt}$ device was free from electroforming. Interestingly, an analog type of RS was observed for the present case irrespective of voltage excitation. In analog-type RS, the current or resistance of the device changes gradually. By contrast, a sudden change in current or resistance is observed in digital RS. These two types of RS have applications in various fields. For instance, analog RS is useful for mimicking biological synaptic properties in neuromorphic computing applications, whereas high-performance nonvolatile memory devices can be developed using digital RS effects ${ }^{37-39}$. One can also utilize analog RS in nonvolatile memory applications $^{11}$. However, it is difficult to mimic biosynaptic properties using the digital RS effect because the natural mechanism of synapses is based on gradual changes in ion transportation $^{40}$. The I-V curves of the $\mathrm{Pt} / \mathrm{NCO} / \mathrm{Pt}$ device depict a hysteresis loop; therefore, memristive properties may exist in the device ${ }^{41}$. We attempted to understand the memristive nature of the device by calculating the device flux, device charge, and charge-flux characteristics from the experimental voltage-dependent $\mathrm{I}-\mathrm{V}$ data ${ }^{42}$.

Figure 4 shows the memristive properties of the $\mathrm{Pt} /$ $\mathrm{NCO} / \mathrm{Pt}$ device. The voltage-dependent time-domain flux properties of the $\mathrm{Pt} / \mathrm{NCO} / \mathrm{Pt}$ device demonstrate symmetric behavior that originates from the symmetric voltage stimulus ${ }^{43}$, as shown in Fig. 4a. On the other hand, the time-domain charge behavior of the device became asymmetric irrespective of the external voltage stimulus (Fig. 4b). The asymmetric behavior originated from the current $(I)$ response of the device and the nature of the hysteresis loop ${ }^{44}$. Here, $A_{1}$ and $A_{2}$ represent the first and final charge values, respectively, whereas the half-period point is designated by the $B_{\mathrm{CW}}$.

Interestingly, the device charge shows tunable behavior as a function of the external voltage stimulus. Therefore, different memristive switching states (resistance) can be achieved by properly modulating the external bias for multilevel RS applications. The combined plot of device charge and flux shows double-valued and nonlinear properties, as shown in Fig. 4c. By definition, an ideal memristor exhibits single-valued and nonlinear properties in the flux and charge plane ${ }^{44}$. Deviations from the ideal definition of a memristor produce a nonideal memristor device or memristive device ${ }^{45}$. Considering the double-valued charge-flux nature, memristive properties dominated in the $\mathrm{Pt} / \mathrm{NCO} / \mathrm{Pt}$ device. The results further suggested that the device can have different low-resistance states (LRSs) and fixed highresistance states (HRSs) (Fig. 4c). A voltage-tunable LRS was observed for the $\mathrm{Pt} / \mathrm{NCO} / \mathrm{Pt}$ memristive device. The turning point $B_{\mathrm{CW}}^{N}$, which is responsible for the chargedriving capability, also shows voltage-tunable properties. The performance parameters of the $\mathrm{Pt} / \mathrm{NCO} / \mathrm{Pt}$ memristive device, i.e., $A_{2}, B_{\mathrm{CW}}^{N}$, and the memristive hysteresis area, are shown in Fig. 4d, e, and f, respectively. In each case, incremental performance was observed with respect to voltage stimulus. An interpretation of the device flux, device charge, and hysteresis area is provided in the supporting information file. 

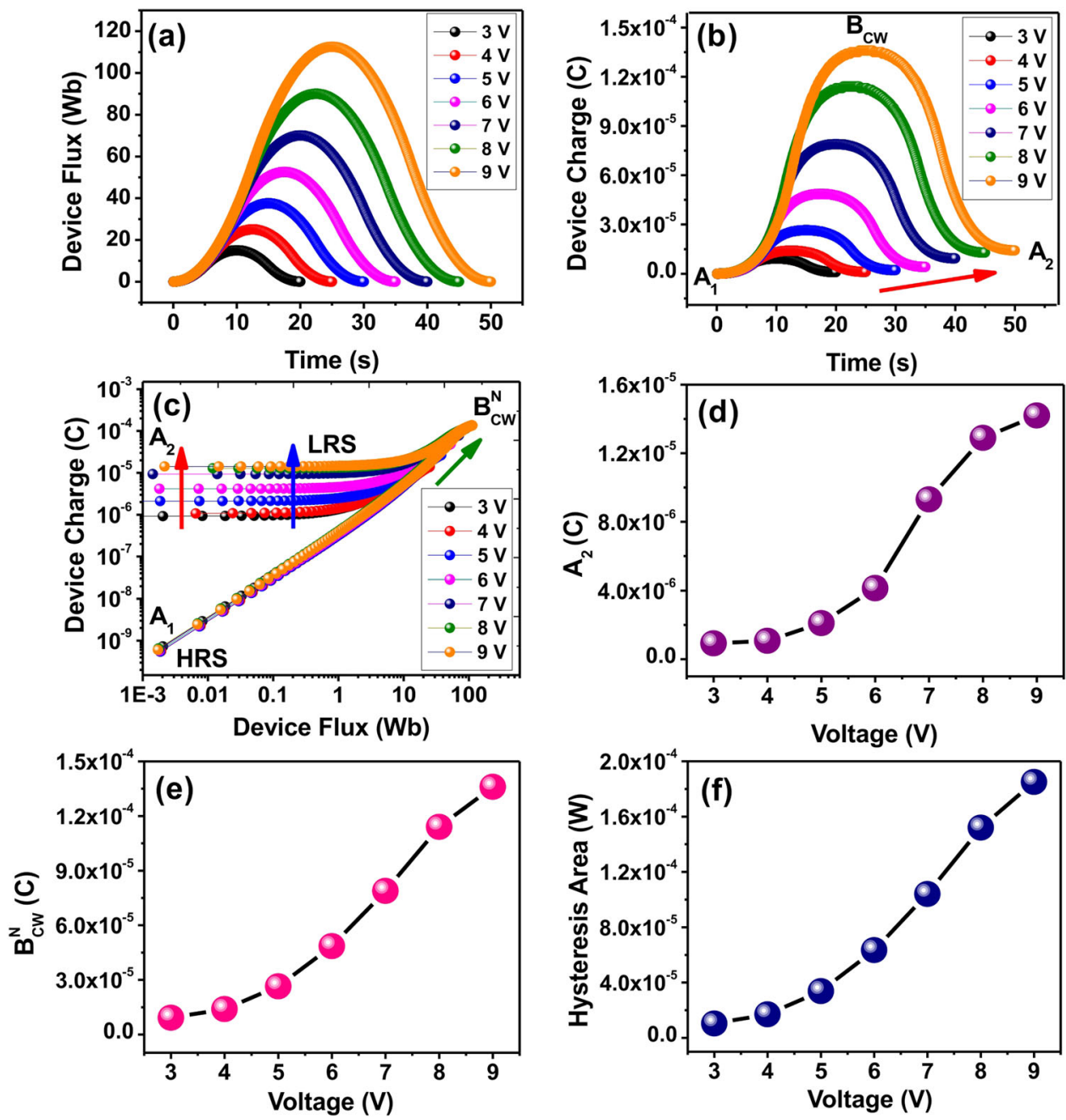

Fig. 4 Different analytical properties of the memristive device based on experimental I-V data. Voltage-dependent a time-domain flux, $\mathbf{b}$ time-domain charge, and $\mathbf{c}$ charge-flux properties of the Pt/NCO/Pt device. Voltage-dependent $\mathbf{d} A 2, e B_{C W}^{N}$, and $\mathbf{f}$ memristive hysteresis area of the Pt/NCO/Pt device.

The conduction mechanism of the $\mathrm{Pt} / \mathrm{NCO} / \mathrm{Pt}$ memristive device was revealed by analyzing the I-V characteristics. Figure S5a (voltage: $+3 \mathrm{~V}$ ) to S5g (voltage: $+9 \mathrm{~V})$ shows the positive-biased double log I-V plots. Figure S6a (voltage: $-3 \mathrm{~V}$ ) to S6g (voltage: $-9 \mathrm{~V}$ ) shows the negative-biased double log $\mathrm{I}-\mathrm{V}$ plots. Among these results, the $\pm V_{\text {STOP }}$ variation ( \pm 3 to $\pm 9 \mathrm{~V}$ ) results were used to understand the charge transport mechanism. The fitting results indicated that the ohmic model fits the low-voltage region ( 0 to $\pm 0.5 \mathrm{~V}$ ) HRS data well for both bias regions, suggesting that the current was proportional to the applied voltage (Fig. S7a, c). In this case, the thermally generated charge carriers took part in conduction. For the high-voltage-region $\left( \pm 0.5\right.$ to $\left.\pm \mathrm{V}_{\mathrm{STOP}}\right)$ HRS data, the current was found to be proportional to the 2.5th power of voltage and was well fitted to the trap-filled limited current model (Fig. S7b, d). In the higher-voltage region, the density of injected charge carriers is higher than that of thermally generated charge carriers. Therefore, a higher current was observed in the high-voltage region than in the low-voltage region. Adj. $R^{2}$ is a statistical measure that is generally used to describe how well a model equation fits the experimental data. For both model-fitting results, the Adj. $R^{2}$ value for each voltage was found to be $>0.99$, suggesting that the model-fitting results explain $99 \%$ of the experimental data well. Considering the electrical fitting results, a plausible RS mechanism of the $\mathrm{Pt} / \mathrm{NCO} / \mathrm{Pt}$ memristive device is shown in Fig. 5. During the synthesis of the $\mathrm{NCO}$ material, a large number of oxygen vacancies could be created due to the solutionprocessable synthesis procedure and the annealing of the thin film. The XPS results (Fig. 2d) confirmed that the $\mathrm{NCO}$ nanomaterial has a large number of oxygen vacancies. These oxygen vacancies are crucial for the RS process. 

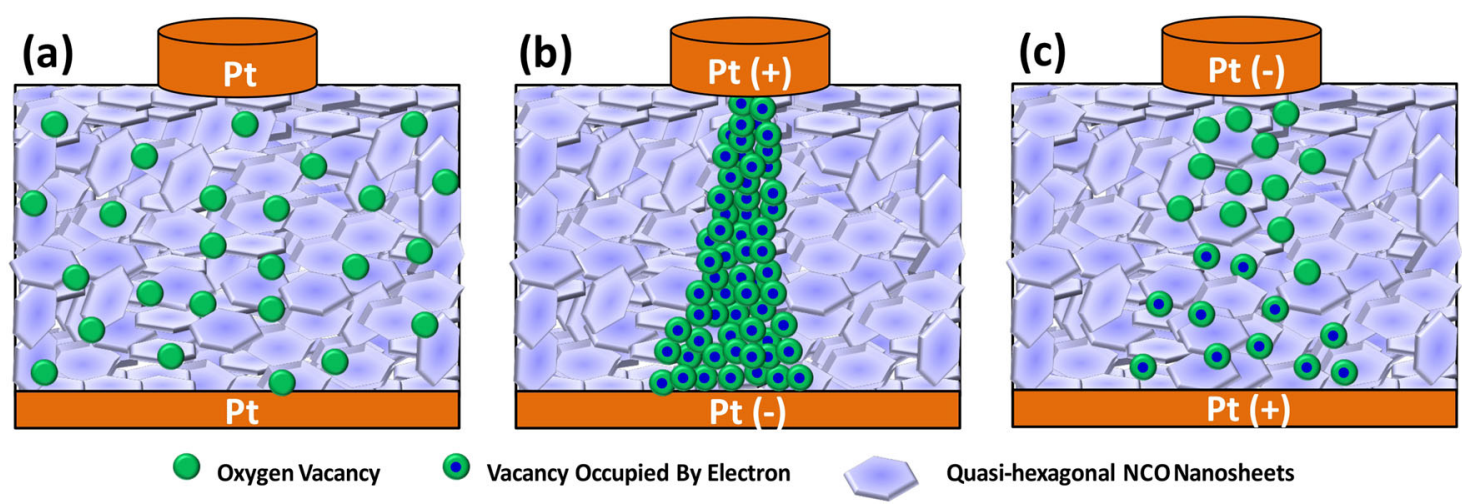

Fig. 5 The filamentary RS mechanism of the device based on electrical fitting results. Plausible RS mechanism of the Pt/NCO/Pt memristive device during a initial state, $\mathbf{b}$ LRS and $\mathbf{c}$ HRS.

When no electric field is applied to the device, the Pt/ $\mathrm{NCO} /$ Pt memristive device shows HRS (Fig. 5a). When the top Pt electrode is subject to a positive bias with respect to the bottom Pt electrode, the conductive filament originates from the bottom Pt electrode and reaches the top Pt electrode. This is because electronic charge carriers are trapped in the oxygen vacancies of $\mathrm{NCO}$ and try to migrate from the bottom electrode to the top electrode. Therefore, the current of the device increases, and the device switches to the LRS (Fig. 5b). The quasi-hexagonal NCO nanosheets have a large surface area, as observed from the FESEM results. Therefore, a direct conductive pathway can be easily formed, which facilitates the formation of a good conductive filament in the NCO switching layer. The trapped carriers can remain in the traps for a long time until the influx of the next electrical stimulus $^{46}$. When the top electrode is subjected to a negative bias, the oxygen vacancies try to migrate towards the top electrode. This results in detrapping of electronic charge carriers, which results in the rupture of the conductive filament (Fig. 5c). Due to the application of negative bias to the top electrode, the device switches to HRS. Cyclic polarity reversal is responsible for the RS process in the $\mathrm{Pt} / \mathrm{NCO} / \mathrm{Pt}$ memristive device.

In a previous experiment, we demonstrated multilevel RS by tuning the external voltage. Multilevel RS is also achieved by adjusting the $\mathrm{CC}$ of the device, and one such investigation is shown in Fig. S8a (voltage: $\pm 3 \mathrm{~V}$ ) to S8i (voltage: $\pm 11 \mathrm{~V})$. In this case, different CCs $\left(10^{-2}-10^{-6} \mathrm{~A}\right.$ ) were applied at different $\pm \mathrm{V}_{\text {STOP }}$ voltages $( \pm 3$ to $\pm 11 \mathrm{~V})$ in the $\mathrm{Pt} / \mathrm{NCO} / \mathrm{Pt}$ memristive device. CC-dependent RS was observed for each $\pm V_{\text {STOP }}$ voltage. However, the device showed fluctuations in RS at \pm 10 and $\pm 11 \mathrm{~V}$. In particular, \pm 3 to $\pm 7 \mathrm{~V}$ showed lower CC $\left(10^{-6} \mathrm{~A}\right)$-dependent RS. In this voltage range, $\mathrm{CC}\left(10^{-2}-10^{-5} \mathrm{~A}\right)$ did not show any

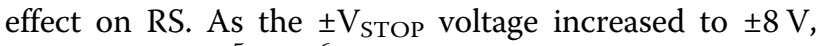
two CCs $\left(10^{-5}-10^{-6} \mathrm{~A}\right)$ showed a dominant effect. In summary, CC-dependent RS was observed at lower
$C C$ values, and $R S$ was independent at higher $C C$ values. Furthermore, we extracted different RS parameters from the experimental data to study the role of $\mathrm{CC}$ in the RS properties of the $\mathrm{Pt} / \mathrm{NCO} / \mathrm{Pt}$ memristive device. The CCdependent $I_{\text {SET }}$ (Fig. 6a), $I_{\text {RESET }}$ (Fig. 6b), $V_{\text {SET }}$ (Fig. 6c), $V_{\text {RESET }}$ (Fig. $6 \mathrm{~d}$ ), $\eta\left(I_{\mathrm{SET}} / I_{\mathrm{CC}}\right)$ (Fig. 6e), and $\eta\left(I_{\mathrm{RESET}} / I_{\mathrm{CC}}\right)$ (Fig. 6f) at different $V_{S T O P}$ voltages were investigated. The results indicate that CC-dependent RS was observed mostly at $10^{-5}$ and $10^{-6} \mathrm{~A}$, while RS was independent at other CC values. The $I_{\mathrm{SET}}$ and $I_{\text {RESET }}$ currents were found to increase as CC changed from $10^{-6} \mathrm{~A}$ and became saturated at higher CCs (Fig. 6a, b).

It is a well-known fact that the thickness of the conductive filament increases as a function of CC, and therefore, a higher current is needed to break the conductive filament ${ }^{24}$. In the same way, $V_{\mathrm{SET}}$ and $V_{\mathrm{RESET}}$ were found to be low at lower CCs and became high at higher CCs (Fig. 6c, d). The high switching voltages were due to the thick conductive filament produced by the greater $\mathrm{CC}$ magnitude. A clear trend in the $\pm \mathrm{V}_{\mathrm{STOP}}$ voltagedependent $I_{\mathrm{SET}}, I_{\mathrm{RESET}}, V_{\mathrm{SET}}$, and $V_{\mathrm{RESET}}$ was observed from the experimental data. These results indicated that the size of the conductive filament was modulated as a function of $\mathrm{CC}^{47}$. The nature of RS properties can be elucidated by the value of $\eta\left(I_{\mathrm{SET}} / I_{\mathrm{CC}}\right)$ or $\left(I_{\mathrm{RESET}} / I_{\mathrm{CC}}\right)$. The CC-dependent $\eta$ values at different $\pm \mathrm{V}_{\text {STOP }}$ voltages are shown in Fig. 6e, f. Symmetric RS showed an $\eta=1$, whereas $\eta$ became $<1$ for asymmetric $\mathrm{RS}^{48}$. In the present case, the magnitude of $\eta$ tended to decrease as CC increased from $10^{-6}-10^{-2} \mathrm{~A}$. On the other hand, $\eta$ approached 1 as the $\pm \mathrm{V}_{\text {STOP }}$ voltage increased from \pm 3 to $\pm 9 \mathrm{~V}$. Given this, asymmetric RS was found at higher CCs and lower $\pm \mathrm{V}_{\text {STOP }}$ voltages.

Figure 7 depicts the multilevel memory properties of the $\mathrm{Pt} / \mathrm{NCO} / \mathrm{Pt}$ memristive device. The CC-based approach was used to measure the multilevel endurance and retention properties of the $\mathrm{Pt} / \mathrm{NCO} / \mathrm{Pt}$ memristive device. For the nonvolatile memory measurements, a voltage pulse 

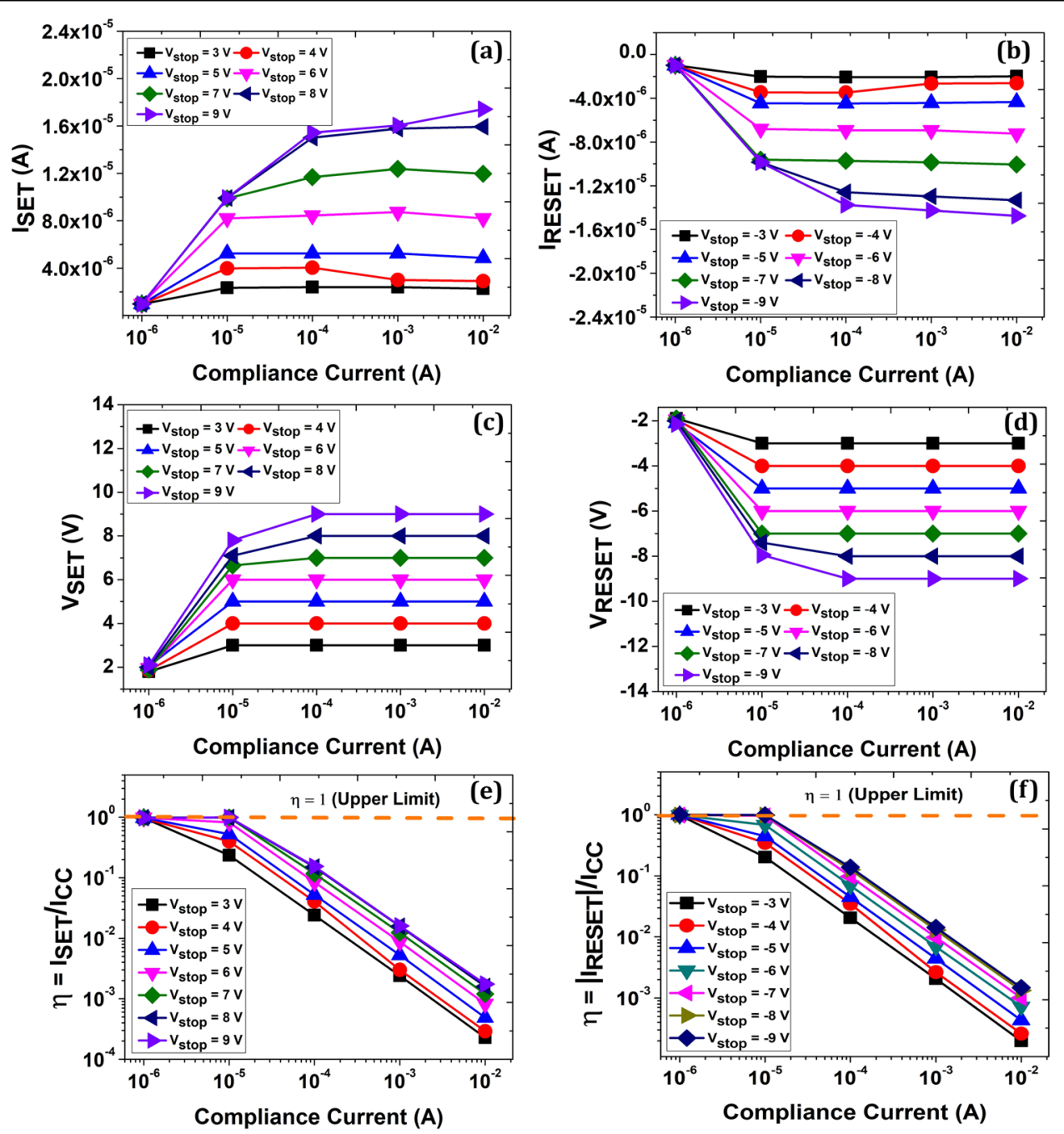

Fig. 6 Different RS parameters of the Pt/NCO/Pt memristive device. Compliance current (CC)-dependent $\mathbf{a} I_{S E T,},\left.\mathbf{b}\right|_{\text {RESET, }} \mathbf{c} V_{S E T}, \mathbf{d} V_{R E S E T}, \mathbf{e} \eta$ ( $I_{S E T} /$ $\left.\mathrm{I}_{\mathrm{CC}}\right)$, and $\mathbf{f} \eta\left(\mathrm{I}_{\mathrm{RESET}} \mid / \mathrm{I}_{\mathrm{CC}}\right)$ at different $\mathrm{V}_{\mathrm{STOP}}$ voltages.
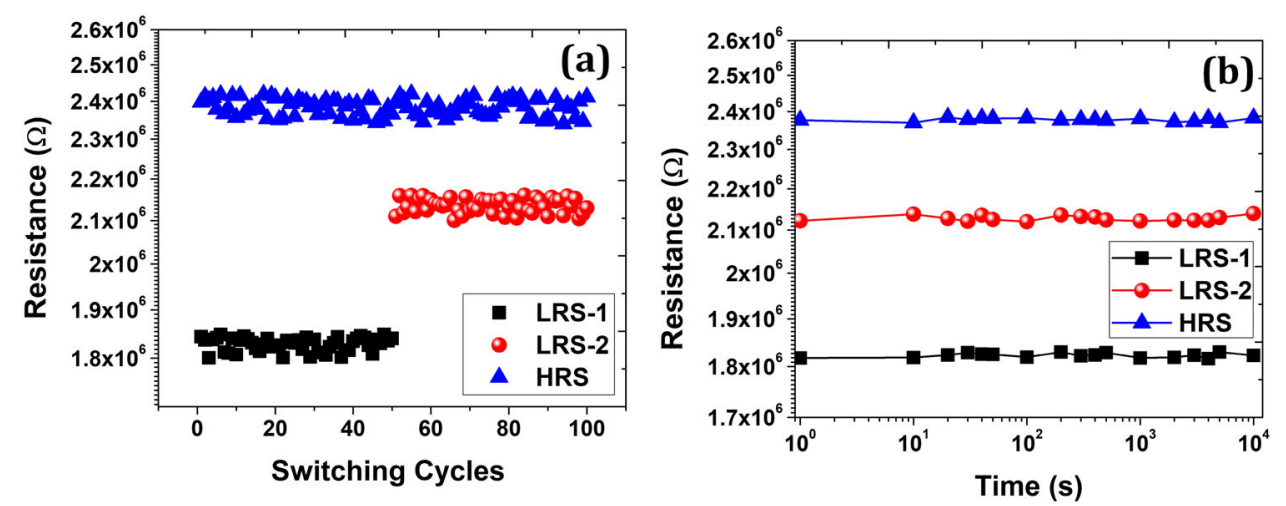

Fig. 7 CC-dependent multilevel RS properties of the device. Multilevel $\mathbf{a}$ endurance and $\mathbf{b}$ retention properties of the Pt/NCO/Pt device. 
with amplitude $\pm 8 \mathrm{~V}$, width $0.5 \mu \mathrm{s}$, and period $2 \mu$ s was applied to the device. The resistance was recorded with a $1 \mathrm{~V}$ read voltage at each switching cycle. Herein, three distinct resistance states were measured by applying a proper CC to the device. Two LRSs $\left(10^{-5} \mathrm{~A} C \mathrm{C}=\mathrm{LRS}-1\right.$ and $\left.10^{-6} \mathrm{~A} C \mathrm{CC}=\mathrm{LRS}-2\right)$ and one HRS were achieved during endurance and retention measurements. Cyclic switching (endurance) showed stable nonvolatile memory behavior during multilevel RS operation (Fig. 7a). Furthermore, the device retained multilevel RS for $10^{4} \mathrm{~s}$, suggesting that it is an overall good candidate for resistive memory applications (Fig. 7b). Size modulation of the conductive filament during $\mathrm{CC}$ operation is a possible reason for multilevel $\mathrm{RS}$ in the $\mathrm{Pt} / \mathrm{NCO} / \mathrm{Pt}$ memristive device $^{24}$. In the literature, many reports have demonstrated that tuning the RESET voltage is a possible solution to achieve multilevel RS in memristive devices ${ }^{23-26,49}$. In the present case, fluctuations were observed during RESETvoltage-based endurance and retention measurements. Therefore, multilevel RS during RESET voltage measurements is not reported in this work.

The neuron and synapse are computational building blocks of the human brain that are mainly responsible for learning and memory. Despite many efforts, it is highly complicated to build or mimic the functionalities of the human brain using traditional von Neumann computers owing to their different memory and processing units. Therefore, a different strategy is needed to mimic brain functionalities to achieve human-like intelligence. Memristive devices inherently possess in-memory computing capabilities $^{5}$, passivity, and two-terminal structures similar to biological neurons ${ }^{50}$. For instance, a sketch of a biological synapse junction sandwiched between pre- and postneurons is shown in Fig. 8a. In biological neurons, the
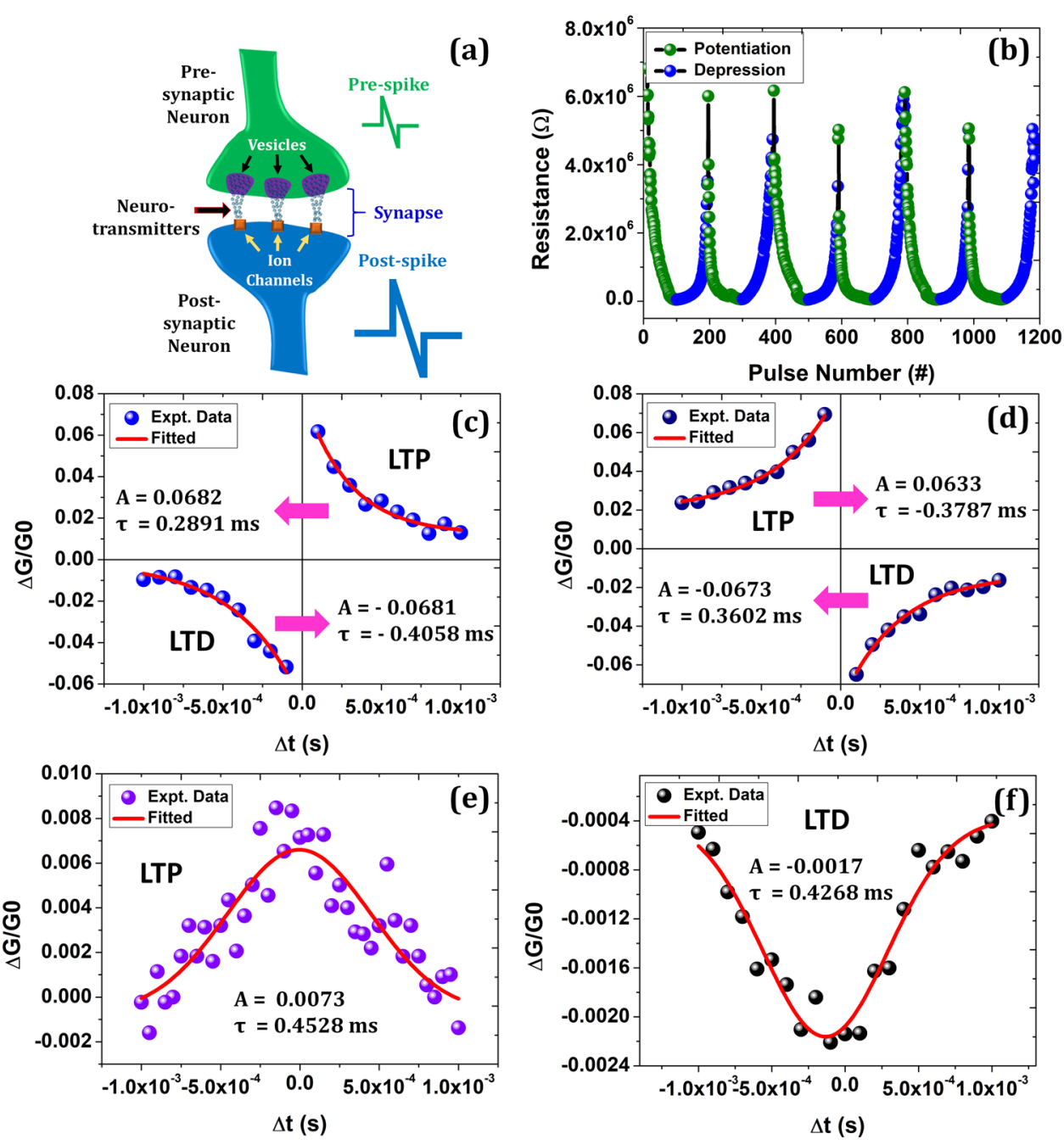

Fig. 8 Different synaptic properties mimicked by the Pt/NCO/Pt memristive device. a Sketch of biological synapse junctions sandwiched between pre- and postneurons. b Potentiation and depression properties of the Pt/NCO/Pt memristive device. c ASH, d ASAH, e SH, and $\mathbf{f} S A H$ learning rules mimicked with the help of the Pt/NCO/Pt memristive device. 
neurotransmitters of a presynaptic neuron are discharged in the synaptic cleft owing to excitation of the prespike or action potential. The neurotransmitters are diffused in the synaptic cleft and received by the receptors of a postsynaptic neuron, resulting in a postsynaptic spike ${ }^{51}$. In a similar vein, the signal from the top electrode (presynaptic terminal) of the memristive device can be transmitted through the active switching layer (synapse) with the help of ionic species and received at the bottom electrode (postsynaptic terminal $)^{52}$. Given this, memristive devices are possible candidates for developing a neuromorphic computing system.

To achieve brain-like computing capabilities, a memristive device should mimic the different forms of synaptic plasticity rules. The potentiation and depression $(\mathrm{P} / \mathrm{D})$ property of biological neurons is a basic form of synaptic plasticity. Generally, the synaptic weights of a neuron can be modulated by the application of consecutive spikes ${ }^{53}$. The synaptic weight-like behavior of the device was realized by measuring I-V hysteresis loops under repeated voltage sweeps (Fig. S9). Here, the device showed increasing and decreasing current responses as a function of repeated voltage sweeps, suggesting modulation of synaptic weights, similar to the behavior of biological synapses $^{54}$. To further demonstrate the resemblance of the $\mathrm{Pt} / \mathrm{NCO} / \mathrm{PT}$ memristive device to a biological synapse, 100 consecutive positive $(+6 \mathrm{~V}, 10 \mathrm{~ms})$ and 100 negative $(-6 \mathrm{~V}, 10 \mathrm{~ms})$ pulses were applied, and the corresponding resistance was measured (read voltage: $1 \mathrm{~V}$ ) using a memristor characterization system (ArC ONE) (Fig. 8b). This platform easily captures the synaptic properties of the tested device upon the selection of predefined or usermodified spike schemes.

The device showed a gradual change in resistance during $\mathrm{P} / \mathrm{D}$ measurements. The device resistance was weakened (increase in conductance) owing to the excitatory pulses, which resulted in potentiation. On the other hand, depression (strengthened device resistance or decrease in conductance) was achieved by the application of inhibitory pulses $^{55}$. The results demonstrated that the $\mathrm{Pt} / \mathrm{NCO} / \mathrm{Pt}$ memristive device can mimic the basic properties $(\mathrm{P} / \mathrm{D})$ of a biological synapse. In addition, complex synaptic weight modification can be achieved by tuning the relative timing between pre- and postsynaptic spikes. In the present case, four different spike-timing-dependent plasticity (STDP) rules [antisymmetric Hebbian (ASH), antisymmetric antiHebbian (ASAH), symmetric Hebbian (SH), and symmetric anti-Hebbian (SAH) learning rules] were mimicked with the help of the Pt/NCO/Pt memristive device. Details of the STDP spike scheme signals used to obtain ASH (Fig. S10a), ASAH (Fig. S10b), SH (Fig. S10c), and SAH (Fig. S10d) are provided in the supporting information. Furthermore, Table S1 summarizes the electrical parameters of the pre- and postsynaptic spike schemes. Herein, the relative time interval between pre- and postsynaptic spikes $(\Delta t)$ was altered to modulate the polarity and amplitude of synaptic weights $(\Delta G / G 0)^{56}$. The optimized spike schemes were applied to the device, and the corresponding synaptic weights were recorded. During measurements, numerous STDP events were applied to the device by changing the $\Delta t$ of the spike with a constant time step, and the resulting $\Delta G / G O$ vs. $\Delta t$ was plotted. In the case of ASH learning, long-term potentiation (LTP) occurred when $\Delta t>0$, and long-term depression (LTD) occurred when $\Delta t<0$, as shown in Fig. 8c. In the case of LTP, the synaptic strength increased, while synaptic strength decreased in LTD. The highest synaptic weight change was observed when $\Delta t$ between paired spikes was very short, and the synaptic weight decreased as $\Delta t$ increased between paired spikes. A similar trend was observed for ASAH learning with synaptic weight modifications with opposite polarity ${ }^{57}$ (Fig. 8d). For SH and $\mathrm{SAH}$ learning rules, the relative timing is very important, but the order is not. Therefore, $\mathrm{SH}$ learning showed good potentiation when $\Delta t \sim 0$. As $\Delta t$ shifted away from 0 , depression dominated (Fig. 8e). The opposite behavior was observed for SAH learning by simply changing the polarity of the spike ${ }^{58}$ (Fig. 8f). For the ASH and ASAH learning rules, the exponential function was well fitted to the experimental data ${ }^{56}$.

$$
\Delta W=A \exp \left(\frac{-\Delta t}{\tau}\right)+\Delta w_{0}
$$

In contrast, the $\mathrm{SH}$ and $\mathrm{SAH}$ learning rules were well fitted to the Gaussian function ${ }^{56}$.

$$
\Delta W=A \exp \left(\frac{-\Delta t^{2}}{\tau^{2}}\right)+\Delta w_{0}
$$

where $\Delta W$ represents the change in synaptic weight, $A$ is a scaling factor, and $\tau$ is a time constant. The fitting parameters are shown in the inset of each figure (Fig. 8c-f). The results demonstrated that the NCO nanosheet-based memristive device realistically mimics the four classical STDP-based Hebbian learning rules. The above results showcase the potential applicability of the $\mathrm{Pt} / \mathrm{NCO} / \mathrm{Pt}$ memristive device in mimicking different synaptic learning rules for brain-like information processing.

\section{Conclusions}

An NCO nanomaterial was synthesized by a coprecipitation method, resulting in a quasi-hexagonal nanosheet morphology. The XPS results confirmed two oxidation states of $\mathrm{Ni}\left(\mathrm{Ni}^{3+}\right.$ and $\left.\mathrm{Ni}^{2+}\right)$ and $\mathrm{Co}\left(\mathrm{Co}^{3+}\right.$ and $\left.\mathrm{Co}^{2+}\right)$. Furthermore, lattice oxygen-, oxygen vacancy-, and surface oxygen-related peaks were also observed. The Pt/NCO/Pt device formed free and voltage-dependent analog-type RS 
properties. The RS properties of the device were found to improve as the applied voltage increased from \pm 3 to $\pm 9 \mathrm{~V}$. Further, symmetric flux and asymmetric charge behavior were observed irrespective of the external voltage stimulus. The device flux and charge showed a double-valued and nonlinear relation, demonstrating the memristive nature of the device. The $A_{2}, B_{\mathrm{CW}}^{N}$, and memristive hysteresis area of the device showed incremental improvements with regard to voltage stimulus. The conduction of the device during HRS was due to the ohmic and trap-filled limited current, whereas the ohmic effect dominated during LRS. Multilevel RS was achieved by adjusting the CC of the device. In particular, a CC-dependent RS was observed at lower CC values, and RS was independent at higher $C C$ values. Three distinct resistance states composed of two LRSs and one HRS were achieved during endurance and retention measurements. Size modulation of the conductive filament during CC operation may cause multilevel RS in the $\mathrm{Pt} /$ $\mathrm{NCO} / \mathrm{Pt}$ memristive device. In the case of biomimicking synaptic properties, the device was able to demonstrate $\mathrm{P} / \mathrm{D}$ properties. Most importantly, the device mimicked different STDP learning rules, including ASH, ASAH, SH, and $\mathrm{SAH}$.

\section{Acknowledgements}

This study was supported by a National Research Foundation of Korea (NRF) grant funded by the Korean government (No. 2016R1A3B 1908249). The authors would like to thank the Samsung Semiconductor Research Center at Korea University for its support.

\section{Conflict of interest}

The authors declare no competing interests.

\section{Publisher's note}

Springer Nature remains neutral with regard to jurisdictional claims in published maps and institutional affiliations.

Supplementary information The online version contains supplementary material available at https://doi.org/10.1038/s41427-021-00286-z.

Received: 24 July 2020 Revised: 9 December 2020 Accepted: 14 December 2020.

Published online: 26 February 2021

\section{References}

1. Acharyya, D., Hazra, A., Dutta, K., Gupta, R. K. \& Bhattacharyya, P. Highly repeatable multilevel resistive switching characteristics of an $\mathrm{Au}^{-T i \mathrm{O}_{2} / \mathrm{Ti}}$ memory device. Semicond. Sci. Technol. 28, 125001 (2013).

2. Houdt, J. Charge-based nonvolatile memory: near the end of the roadmap? Curr. Appl. Phys. 11, e21-e24 (2011).

3. Seok, J. Y. et al. A review of three-dimensional resistive switching cross-bar array memories from the integration and materials property points of view. Adv. Funct. Mater. 24, 5316-5339 (2014).

4. Tian, J. et al. Nanoscale topotactic phase transformation in $\mathrm{SrFeO}_{x}$ epitaxial thin films for high-density resistive switching memory. Adv. Mater. 31, 1903679 (2019).

5. Ielmini, D. \& Wong, H. In-memory computing with resistive switching devices. Nat. Electron. 1, 333-343 (2018).

6. Jordan, M. I. \& Mitchell, T. M. Machine learning: trends, perspectives, and prospects. Science 349, 255-260 (2015).
7. Ielmini, D. Brain-inspired computing with resistive switching memory (RRAM): devices, synapses and neural networks. Microelectron. Eng. 19, 44-53 (2018).

8. Zhang, W. et al. Analog-type resistive switching devices for neuromorphic computing. Phys. Status Solidi (RRL)-Rapid Res. Lett. 13, 1900204 (2019).

9. Wang, Z. et al. Engineering incremental resistive switching in $\mathrm{TaO}_{x}$ based memristors for brain-inspired computing. Nanoscale 8, 14015-14022 (2016).

10. Wan, T. et al. Digital to analog resistive switching transition induced by graphene buffer layer in strontium titanate based devices. J. Colloid Interface Sci. 512, 767-774 (2018).

11. Shi, T., Yang, R. \& Guo, X. Coexistence of analog and digital resistive switching in BiFeO3-based memristive devices. Solid State Ion. 296, 114-119 (2016).

12. Jung, $\mathrm{H}$. et al. Resistive switching characteristics of $\mathrm{ZnO}$ nanoparticles layer-bylayer assembly based on cortisol and its antibody immune binding. J. Ind. Eng. Chem. 78, 66-72 (2019).

13. Lee, S., Yun, K. \& Kim, D. Electroforming-less and multi-level resistive switching characteristics in tungsten oxide thin film. Thin Solid Films 674 91-96 (2019)

14. Patil, A. A., Wagh, S. V., Dongale, T. D. \& Kim, D. K. Electrospun 1D f-MWCNTs$\mathrm{TiO}_{2}$ composite nanofibers for resistive memory and synaptic learning applications. Mater. Lett. 280, 128587 (2020).

15. Li, Y. et al. Analog and digital bipolar resistive switching in solutioncombustion-processed NiO memristor. ACS Appl. Mater. Interface 10, 24598-24606 (2018)

16. $\mathrm{Hu}, \mathrm{W}$. et al. Opportunity of spinel ferrite materials in nonvolatile memory device applications based on their resistive switching performances. J. Am. Chem. Soc. 134, 14658-14661 (2012)

17. $\mathrm{Li}, \mathrm{Y}$., Han, $\mathrm{X} ., \mathrm{Yi}, \mathrm{T}$., He, Y. \& Li, X. Review and prospect of $\mathrm{NiCO}_{2} \mathrm{O}_{4}$-based composite materials for supercapacitor electrodes. J. Energy Chem. 31, 54-78 (2019).

18. Zhang, G. \& Lou, X. General solution growth of mesoporous $\mathrm{NiCO}_{2} \mathrm{O}_{4}$ nanosheets on various conductive substrates as high-performance electrodes for supercapacitors. Adv. Mater. 25, 976-979 (2013).

19. Lim, D. et al. Spinel-type $\mathrm{NiCO}_{2} \mathrm{O}_{4}$ with abundant oxygen vacancies as a highperformance catalyst for the oxygen reduction reaction. Int. J. Hydrog. Energy 44, 23775-23783 (2019)

20. Feng, $X$. et al. Controllable synthesis of porous $\mathrm{NiCO} \mathrm{O}_{4} / \mathrm{NiO} / \mathrm{CO}_{3} \mathrm{O}_{4}$ nanoflowers for asymmetric all-solid-state supercapacitors. Chem. Eng. J. 368, 51-60 (2019).

21. Bhagwan, J., Nagaraju, G., Ramulu, B., Sekhar, S. C. \& Yu, J. S. Rapid synthesis of hexagonal $\mathrm{NiCO}_{2} \mathrm{O}_{4}$ nanostructures for high-performance asymmetric supercapacitors. Electrochim. Acta 299, 509-517 (2019).

22. Venkatachalam, V., Alsalme, A., Alghamdi, A. \& Jayavel, R. Hexagonal-like $\mathrm{NiCO}_{2} \mathrm{O}_{4}$ nanostructure based high-performance supercapacitor electrodes. lonics 23, 977-984 (2017).

23. Prakash, A. \& Hwang, H. Multilevel cell storage and resistance variability in resistive random access memory. Phys. Sci. Rev. 1, 1-16 (2016).

24. Prakash, A. et al. Demonstration of low power 3-bit multilevel cell characteristics in a TaO ${ }_{x}$-based RRAM by stack engineering. IEEE Electron Device Lett. 36, 32-34 (2014)

25. $L v, H$. et al. Evolution of conductive filament and its impact on reliability issues in oxide-electrolyte based resistive random access memory. Sci. Rep. 5, 7764 (2015).

26. Kim, S. T. \& Cho, W. J. Improvement of multi-level resistive switching characteristics in solution-processed $\mathrm{AlO}_{x}$-based non-volatile resistive memony using microwave irradiation. Semicond. Sci. Technol. 33, 015009 (2017).

27. Pan, L., Zhang, Y. \& Shi, C. E. Synthesis of quasi-hexagonal $\mathrm{Ag} / \mathrm{NiCO}_{2} \mathrm{O}_{4}$ nanosheets and their photocatalytic and antibacterial properties. J. Iran. Chem. Soc. 17, 151-157 (2020)

28. Zhang, B. et al. Rapid synthesis of hexagonal mesoporous structured $\mathrm{NiCO}_{2} \mathrm{O}_{4}$ via rotary evaporation for high performance supercapacitors. Ceram. Int. $\mathbf{4 4}$ 8695-8699 (2018)

29. Balasubramanian, P. et al. Oxygen vacancy confined nickel cobaltite nanostructures as an excellent interface for the enzyme-free electrochemical sensing of extracellular $\mathrm{H} 2 \mathrm{O} 2$ secreted from live cells. N. J. Chem. 44, 14050-14059 (2020).

30. Zhang, Q. et al. Growth of hierarchical $3 \mathrm{D}$ mesoporous $\mathrm{NiSi} \times \mathrm{NiCO}_{2} \mathrm{O}_{4}$ core/ shell heterostructures on nickel foam for lithium-ion batteries. ChemSusChem 7, 2325-2334 (2014). 
31. Yuan, C. et al. Polymer-assisted synthesis of a 3D hierarchical porous networklike spinel $\mathrm{NiCO}_{2} \mathrm{O}_{4}$ framework towards high-performance electrochemical capacitors. J. Mater. Chem. A 1, 11145-11151 (2013).

32. Wei, S. et al. Preparation of hierarchical core-shell C@NiCo $\mathrm{O}_{4} @ \mathrm{Fe}_{3} \mathrm{O}_{4}$ composites for enhanced microwave absorption performance. Chem. Eng. J. 314, 477-487 (2017).

33. Huang, L., Zhang, W., Xiang, J. \& Huang, Y. Porous $\mathrm{NiCO}_{2} \mathrm{O}_{4} / \mathrm{C}$ nanofibers replicated by cotton template as high-rate electrode materials for supercapacitors. J. Materiomics 2, 248-255 (2016)

34. Munjal, S. \& Khare, N. Electroforming free controlled bipolar resistive switching in $\mathrm{Al} / \mathrm{CoFe}_{2} \mathrm{O}_{4} / \mathrm{FTO}$ device with self-compliance effect. Appl. Phys. Lett. 112 073502 (2018)

35. Veeramani, $\mathrm{V}$. et al. $\mathrm{NiCO}_{2} \mathrm{O}_{4}$-decorated porous carbon nanosheets for high-performance supercapacitors. Electrochim. Acta 247, 288-295 (2017).

36. Patil, S. R. et al. Solution-processable $\mathrm{ZnO}$ thin film memristive device for resistive random access memory application. Electronics 7, 445 (2018).

37. Sharath, S. U. et al. Towards forming-free resistive switching in oxygen engineered $\mathrm{HfO}_{2-\mathrm{x}}$. Appl. Phys. Lett. 104, 063502 (2014).

38. Kim, H. J. et al. Digital versus analog resistive switching depending on the thickness of nickel oxide nanoparticle assembly. RSC Adv. 3, 20978-20983 (2013).

39. Yen, H. J. \& Liou, G. S. Solution-processable triarylamine-based highperformance polymers for resistive switching memory devices. Polym. J. 48 117-138 (2016).

40. Sokolov, A. S., Jeon, Y. R., Kim, S., Ku, B. \& Choi, C. Bio-realistic synaptic characteristics in the cone-shaped ZnO memristive device. NPG Asia Mater. 11, 5 (2019).

41. Biolek, Z. \& Biolek, D. How can the hysteresis loop of the ideal memristor be pinched? IEEE Trans. Circuits Syst. I/ Express Briefs 61, 491-495 (2014).

42. Du, N. et al. Practical guide for validated memristance measurements. Rev. Sci. Instrum. 84, 023903 (2013).

43. Patil, A. P. et al. Tuning the analog and digital resistive switching properties of $\mathrm{TiO}_{2}$ by nanocompositing Al-doped ZnO. Mater. Sci. Semicond. Process. 115, $105110(2020)$

44. Chua, L. Memristor-the missing circuit element. IEEE Trans. Circuit Theory 18, 507-519 (1971).
45. Chua, L. \& Kang, S. Memristive devices and systems. Proc. IEEE 64, 209-223 (1976).

46. Das, N. C., Oh, S. I., Rani, J. R., Hong, S. M. \& Jang, J. H. Multilevel bipolar electroforming-free resistive switching memory based on silicon oxynitride. Appl. Sci. 10, 3506 (2020).

47. Vishwanath, S., Woo, H. \& Jeon, S. Enhancement of resistive switching properties in $\mathrm{Al}_{2} \mathrm{O}_{3}$ bilayer-based atomic switches: multilevel resistive switching. Nanotechnology 29, 235202 (2018).

48. Ambrogio, S., Balatti, S., Choi, S. \& lelmini, D. Impact of the mechanical stress on switching characteristics of electrochemical resistive memory. Adv. Mater. 26 3885-3892 (2014)

49. Wu, C., Li, F., Zhang, Y., Guo, T. \& Chen, T. Highly reproducible memory effect of organic multilevel resistive-switch device utilizing graphene oxide sheets/ polyimide hybrid nanocomposite. Appl. Phys. Lett. 99, 042108 (2011).

50. Jo, S. H. et al. Nanoscale memristor device as synapse in neuromorphic systems. Nano Lett. 10, 1297-1301 (2010).

51. Kuzum, D., Yu, S. \& Wong, H. Synaptic electronics: Materials, devices and applications. Nanotechnology 24, 382001 (2013).

52. Indiveri, G., Linares-Barranco, B., Legenstein, R., Deligeorgis, G. \& Prodromakis, T. Integration of nanoscale memristor synapses in neuromorphic computing architectures. Nanotechnology 24, 384010 (2013).

53. Qu, B. et al. Synaptic plasticity and learning behavior in transparent tungsten oxide-based memristors. Mater. Des. 129, 173-179 (2017).

54. Wu, C., Kim, T. W., Choi, H. Y., Strukov, D. B. \& Yang, J. J. Flexible threedimensional artificial synapse networks with correlated learning and trainable memory capability. Nat. Commun. 8, 752 (2017).

55. Yang, C. S. et al. Electrochemical-reaction-induced synaptic plasticity in $\mathrm{MoO}_{\mathbf{x}}$ based solid state electrochemical cells. Phys. Chem. Chem. Phys. 19, 4190-4198 (2017).

56. Li, Y. et al. Ultrafast synaptic events in a chalcogenide memristor. Sci. Rep. $\mathbf{3}$ 1619 (2013)

57. Majumdar, S., Tan, H., Qin, Q. H. \& van Dijken, S. Energy-efficient organic ferroelectric tunnel junction memristors for neuromorphic computing. Adv. Electron. Mater. 5, 1800795 (2019).

58. Jiang, C. et al. Efficient two-terminal artificial synapse based on a network of functionalized conducting polymer nanowires. J. Mater. Chem. C 7, 9933-9938 (2019). 